\title{
Microwave sensors for detection of wild animals during pasture mowing
}

\author{
A. Patrovsky ${ }^{1,2}$ and E. M. Biebl ${ }^{2}$ \\ ${ }^{1}$ Technische Universität München, Fachgebiet Höchstfrequenztechnik, Arcisstraße 21, 80333 München, Germany \\ ${ }^{2}$ now at: Ecole Polytechnique, University of Montreal, C.P. 6079 Montreal, H3C 3A7 Canada
}

\begin{abstract}
More than 400000 wild animals are killed or severely injured every year during spring time pasture mowing. Conventional methods for detection and removal or expulsion of animals before mowing are either inefficient or very time-consuming. The first really working method is based on a pyro-detector which senses the temperature contrast between the animals body and the surrounding pasture. Unfortunately, the detection reliability of this sensor decreases with increasing ambient temperature and strong sunlight, i.e. for typical weather conditions, when pasture is mowed, especially around noon.

In this paper, a detector is presented that exhibits complementary behaviour. It works best during dry conditions (i.e. around noon), but has a tendency to false alarms when dew is present (i.e. morning and evening). The sensor is based on a commercial, low-cost Doppler module at $24 \mathrm{GHz}$. It senses the difference of radar cross section between the animals body (high water content, specular reflection) and the pasture (low water content, diffuse reflection). The signal is analysed by means of a non-linear Wigner time-frequency transformation. Experimental results are presented for a laboratory setup as well as for measurement in actual springtime pasture. The results prove that a microwave sensor is capable of reliably detecting animals of the size of a fawn even if it is covered by a layer of pasture.
\end{abstract}

\section{Introduction}

More than 400000 wild animals are killed or severely injured every year during spring time pasture mowing for hay and silage production in the western part of Germany only. Fawns, leverets (young hares) and clutches of birds breeding in the meadows are hidden in the pasture and cannot be detected visually by an operator of a mowing-machine. Thus, several strategies and methods have been developed in the past to find and remove or expel these animals before and during mowing:

Correspondence to: $\mathrm{E} . \mathrm{M}$. Biebl

(biebl@tum.de)
- Scaring away: One day before mowing the meadow some unusual disturbance is made, e.g. by loud noise, scarecrows or dogs. During night the roe deer leads her fawns hidden in this meadow into the forest or another meadow. This method is not very reliable (especially in densely populated areas wild animals are very tolerant to disturbance) and requires careful planning of the mowing. Obviously, this method does not prevent the destruction of clutches.

- Searching with a hunting dog: In the morning before mowing the meadow is searched by use of a well-trained hunting dog. This method is extremely time-consuming requiring much more time than the mowing. Thus, it is restricted to relatively small meadows. Clutches are easily removed, but it is difficult to prevent the return of found and removed fawns into the meadow.

- Mechanical solutions: Several kinds of mechanical protection systems like large combs in front of the mower have been developed and investigated. Unfortunately, these devices tend to impede the mowing machine or to pass over the cowering animal.

In conclusion, these conventional methods are very timeconsuming or ineffective. Recently, a system based on thermal sensors (Fig. 1) has been developed at the German Aerospace Center (DLR, Oberpfaffenhofen, Germany) in cooperation with the company ISA, Weiden, Germany (Haschberger, 1996) . At a transverse aluminium bar carried by a person ten pyro-sensors are mounted. Each sensor scans a stripe of the meadow (about $80 \mathrm{~cm}$ wide) and detects the thermal contrast between the animal's body or the clutch and the surrounding pasture. Many of these systems are operated since years and exhibit a high, documented detection rate. However, since pasture is usually mowed during sunshine, the thermal contrast decreases during the course of a day leading to a significant number of false alarms. For an automated detection system, e.g. mounted at a mowing machine, only a very small rate of false alarms can be tolerated.

Thus, an additional microwave sensor based on a commercial $24 \mathrm{GHz}$ Doppler radar module has been developed that detects the contrast regarding reflectivity and radar cross 


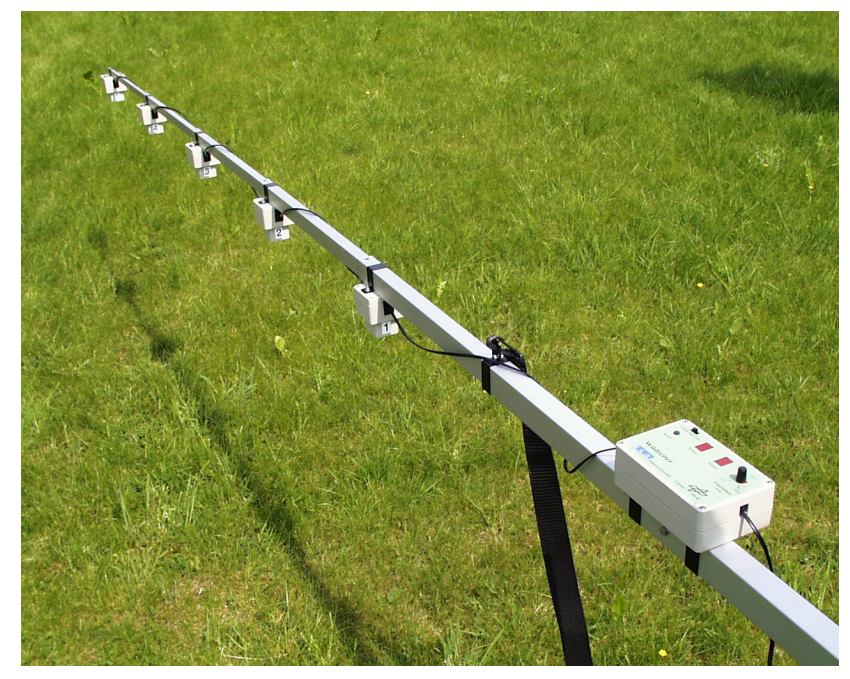

Fig. 1. Portable detection system based on eight pyro-sensors.

section between the animal's body and the pasture. The typical radar response of the convex, due to the water content highly reflective body differs significantly from the "noisy" response of the pasture. Investigation of the time-frequency spectrum of the Doppler signal showed that moving the sensor over the body results in a chirped Doppler signal that can reliably be detected by use a nonlinear Wigner transformation. The microwave wildlife sensor was successfully tested in the laboratory as well as in real pasture.

\section{Doppler radar sensor}

Figure 2 shows the proposed setup of an automated detection system mounted at a mowing machine. A set of microwave sensors is mounted at a standard tool bar (e.g. carrier of a fertilizer spreader) scanning the stripe of pasture to be mowed next. If an animal is detected, the operator is noticed and the position is marked on a GPS based map. When the next stripe is mowed, the operator can either remove the animal or simply go around leaving out a small spot of pasture.

In the past it has been proven that even simple Doppler sensors are capable of performing complex sensing task, if suitable signal processing is applied to the Doppler signal (e.g. Biebl, 1999; Rasshofer, 1999). The Doppler radar sensor employed in our investigations is a commercial, low-cost module based on hollow-guide technology. The signal of a $24 \mathrm{GHz}$ Gunn oscillator is partly radiated via a horn antenna (about $5 \mathrm{~mW}$ of output power) and partly used as LO of a Schottky diode mixer (see Fig. 3). Using this mixer the received Doppler-shifted signal is down-converted to baseband. The down-converted signal is filtered by a band-pass $(10 \mathrm{~Hz}-1 \mathrm{kHz})$, amplified by $70 \mathrm{~dB}$ and fed into a standard PC soundcard for A/D-conversion and processing. Dopplermodule, analogue processing (filter and amplifier) and some circuitry for power supply was assembled in a rigid housing

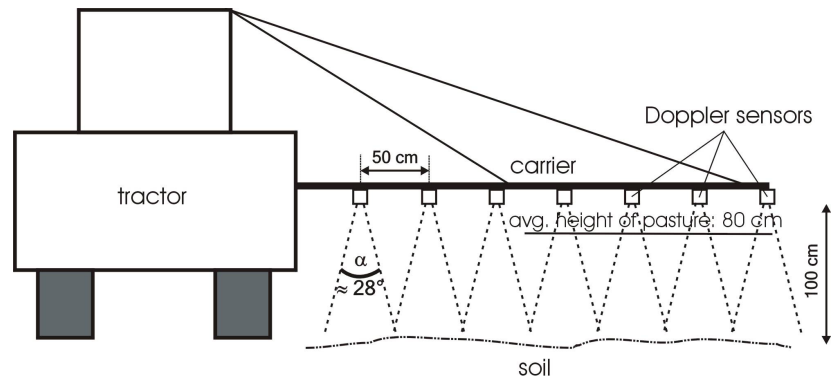

Fig. 2. A proposed microwave detection system mounted at a mowing machine.

including a protective dielectric cover for the horn antenna as shown in Fig. 4.

The geometry of the horn antenna leads to a beam-width of $25^{\circ}$ and $28^{\circ}$ in E- and H-plane, respectively. We obtained best results when the axis of the horn antenna was inclined to an angle of $20^{\circ}$ with respect to the vertical direction (see Fig. 3). Our experiments showed that a reliable recognition of an animal's body can be obtained by means of the typical sequence of the specular reflection of the radar signal from the body. When the sensor goes over the body at constant speed it will initially detect diffuse reflection. In this case, the Doppler signal look like noise with relatively small amplitude. As soon as the beam hits the animal's body specular reflection results in a sharp increase of the amplitude. According to the radiation pattern the amplitude increases further, then decreases and, eventually, only noise from the diffuse reflection is detected. In addition, the frequency of the Doppler signal changes in time: Since the Doppler frequency depends of the effective inclination angle, i.e. the angle of the line between the point of specular reflection and the sensor's phase centre, the Doppler frequency decreases during the movement of the sensor of the animal's body. In other words, a down-chirped time-frequency spectrum of the Doppler signal is expected.

\section{Signal analysis}

Several possibilities can be taken into account in order to analyze the Doppler signal caught by the radar sensor.

An evaluation in the time domain by cross correlation with sample signal sequences has been investigated in the laboratory. Results showed however that this method was too sensitive on specific objects. Analysis exclusively in the frequency domain is not sufficient, because time information is needed for location of the object to be detected. Socalled joint time-frequency analysis (Cohen, 1995; Quian et al., 1996) is used in these cases where time varying spectra emerge.

As it is known from the uncertainty principle, time and frequency resolution cannot be infinite at the same time. The time-bandwidth-product is a measure for the overall resolution if a signal or signal section in the time-frequency-plane. 


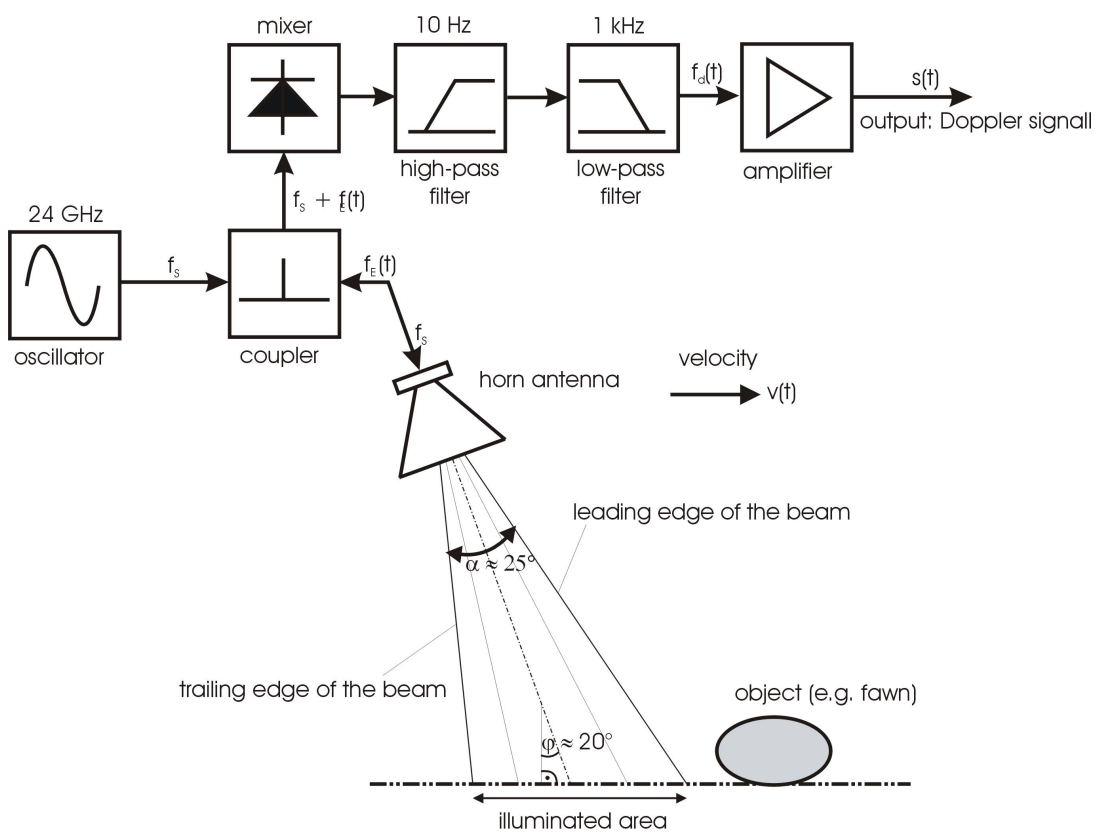

Fig. 3. Doppler radar front-end of the microwave sensor.

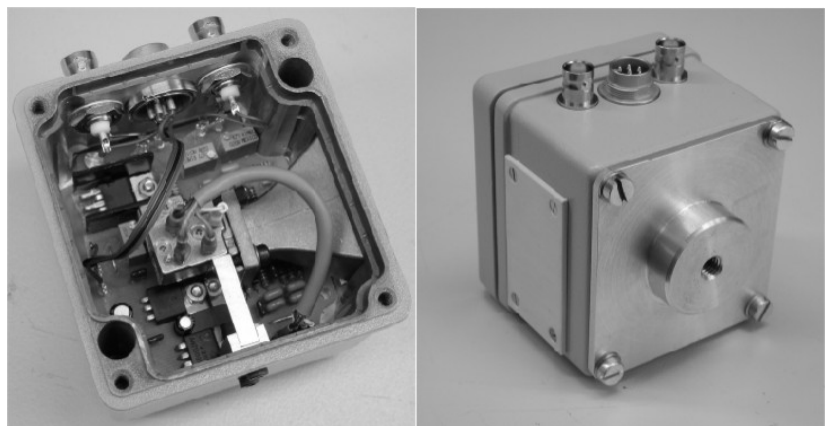

Fig. 4. Photographs of the microwave sensor.

It should be mentioned that the time-frequency characteristics of a signal section differs from these of the entire signal.

The simplest joint time-frequency analysis method is the short time Fourier transformation (STFT) which is basically a Fourier transformation of consecutive sections of a time signal limited by a time window. The shape of this window has to be chosen carefully in order to get good confinement in the frequency domain. The Hanning window was used for further investigations. A compromise has to be found for the window width, which determines the resolution in the time and frequency domain.

Mathematically, the STFT is given by

$\operatorname{STFT}_{x}(t, f)=\int_{-\infty}^{+\infty} \gamma\left(t^{\prime}-t\right) x\left(t^{\prime}\right) e^{-j 2 \pi f t^{\prime}} d t^{\prime}$

where $x(t)$ is a real time signal and $\gamma\left(t^{\prime}-t\right)$ is the time window around $t$.
Advantages of the STFT are simplicity and linearity as well as the availability of efficient algorithms, namely the Fast Fourier Transform (FFT). However, by applying a window on the time signal, the time-bandwidth-product of the resulting signal section degrades dramatically, so that the overall resolution was found not to be sufficient for radar detection of animals.

Wavelet transform has been considered as well, which uses scaled, time-limited wavelets as basis functions instead of sine or cosine functions. As a result of the scaling, the frequency coefficients are calculated more often, but the absolute bandwidth rises with frequency. This may be an advantage for broadband signals, but if we assume a quasiconstant velocity of the vehicle, the Doppler signal bandwidth is rather narrow and convincing improvements cannot be expected from this technique.

A nonlinear transformation, the so-called Wignerdistribution (WD), showed to have favorable properties concerning the resolution problem.

Detailed motives and derivation of the WD can be found in (Quian, 1996). An intermediate step in the derivation is the ambiguity function, which can be regarded as a parallel autocorrelation function in time and frequency domain. It represents an important valuation criterion in radar engineering. After transfer from the relative to the absolute timefrequency-plane, the Wigner distribution (WD) of a time signal $x(t)$ is written as

$$
W_{x x}(t, f)=\int_{-\infty}^{+\infty} x^{*}\left(t-\frac{\tau}{2}\right) \cdot x\left(t+\frac{\tau}{2}\right) e^{-j 2 \pi f \tau} d \tau .
$$

The $x x$ index is added in order to distinguish the autoWD from the likewise existing cross-WD of two different 


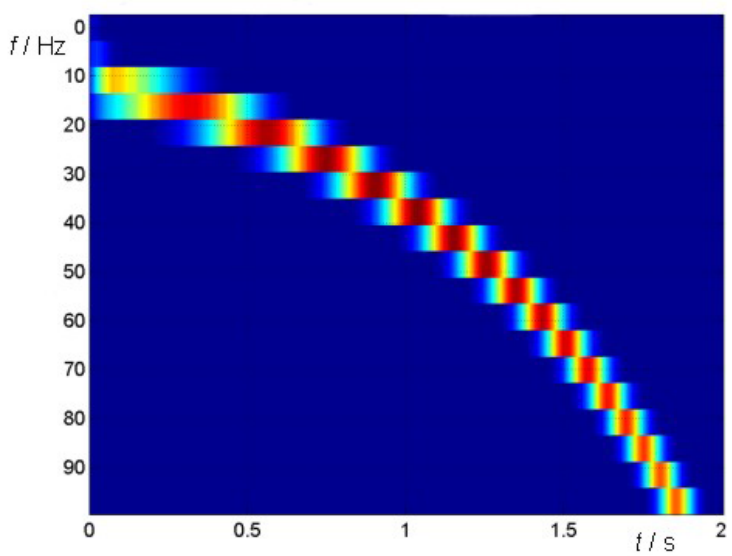

Fig. 5. Time-frequency distribution $|\mathrm{STFT}|^{2}$ of a frequency chirp calculated by means of short-time Fourier transform (frequency resolution: $5.4 \mathrm{~Hz}$; time resolution: $0.186 \mathrm{~s}$ ).

signals. Even if the conjugate complex signal is not needed for real signals, it is added here for later considerations. Some characteristics of the WD are listed in (Quian, 1996). It can be seen that the WD is a nonlinear, or more precisely, a bilinear transformation. Of course, as every nonlinear transformation, it will exhibit the really existing peaks in the time-frequency-plane, but also sum and difference terms. If for instance the time-frequency distribution of a signal with frequency components $f_{1}$ and $f_{2}$ is needed, the WD will also produce peaks at $\left(f_{1}-f_{2}\right) / 2$ and $\left(f_{1}+f_{2}\right) / 2$. Ville proposed to use the analytic signal to get rid of the $\left(f_{1}-f_{2}\right) / 2$ cross-term at low frequencies. The analytic signal is mathematically described by the Hilbert-transformation and serves to eliminate the negative frequency components of a real time signal. The Wignertransformation of the analytic signal is called Wigner-Villedistribution (WVD).

Up to now the transformation is carried out over an infinite time interval. It can be shown that, in this case, the result has the same time-bandwidth-product as the original signal. Due to limited computational resources, the time interval must be limited by a time window. Furthermore, this implicates reduced cross-terms, which arise from non-linearity. In comparison to the STFT, however, the length of the window can be chosen freely without reducing the time resolution. With a window applied, the transformation is called PseudoWigner-Ville-transformation (PVWT).

$$
P W V_{x x}(t, f)=\int_{-\infty}^{+\infty} \gamma(\tau) \cdot x_{a}^{*}\left(t-\frac{\tau}{2}\right) \cdot x_{a}\left(t+\frac{\tau}{2}\right) e^{-j 2 \pi f \tau} d \tau
$$

$\gamma(\tau)$ represents the time window function and $x_{a}$ the analytic signal.

In order to become aware of the benefits of the PWVT, it was compared to the STFT on the basis of a down-chirped signal from an audio signal generator. Figure 5 shows the

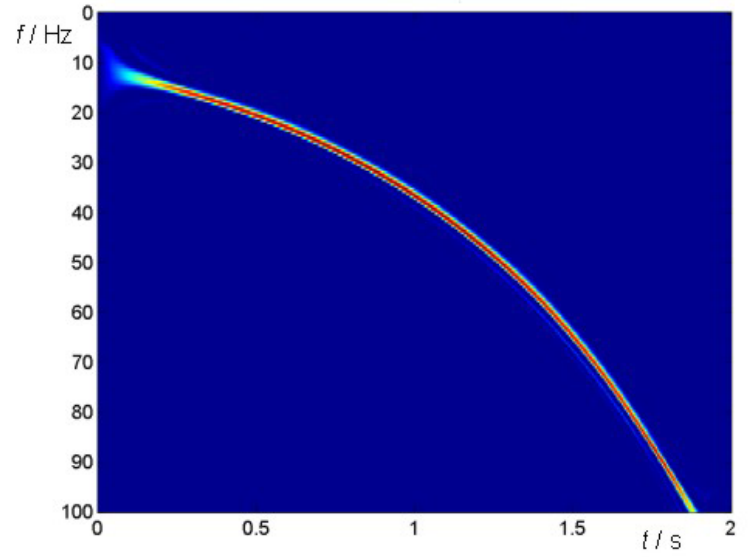

Fig. 6. Time-frequency distribution of a frequency chirp calculated by means of pseudo-Wigner-Ville-transform PWVT (frequency resolution: $0.49 \mathrm{~Hz}$ ).

course of the spectral peak for the STFT. The frequency resolution is noticeably bad and the time window cannot be chosen narrower as this would result in a too heavily blurred spectrum. The course of the spectrum peak calculated by the PWVT is presented in Fig. 6. It is clearly superior in terms of frequency resolution and the noisy curve before and after the object slope also indicates an improved time resolution. A disadvantageous property of the Wigner transformation is the generation of spurious cross-terms next to the proper energy distribution, which are a consequence of the nonlinear operation. These can cause severe perturbations during radar detection, if reflections from the object are weak. Another drawback is the missing possibility of compensation for mechanical oscillations after the transformation. In contrast to linear transformations it is not feasible to use an extra sensor to catch only the oscillation and to subtract this portion of the Doppler signal afterwards.

\section{Evaluation in the laboratory}

The sensor hardware and the analogue and digital signal processing was developed and evaluated in a laboratory setup shown in Fig. 7. The sensor was mounted beneath a large model train running on wooden bar forth and back at constant, but adjustable speed. As a lab-model of pasture we grew a stripe of grass and as a lab model of a fawn we used a fabric-covered hot-water bottle made of rubber. Comparison with a real animal of similar size showed that we obtain from these models very similar signals for both the pyro-sensor and the microwave sensor.

The Doppler baseband signal, obtained from the radar sensor, lies in a frequency range from $10 \mathrm{~Hz}$ to $500 \mathrm{~Hz}$, depending on the antenna elevation angle and velocity of the vehicle. For this reason an ordinary PC sound card was used as $\mathrm{A} / \mathrm{D}$ converter. The sampling rate should not be chosen 


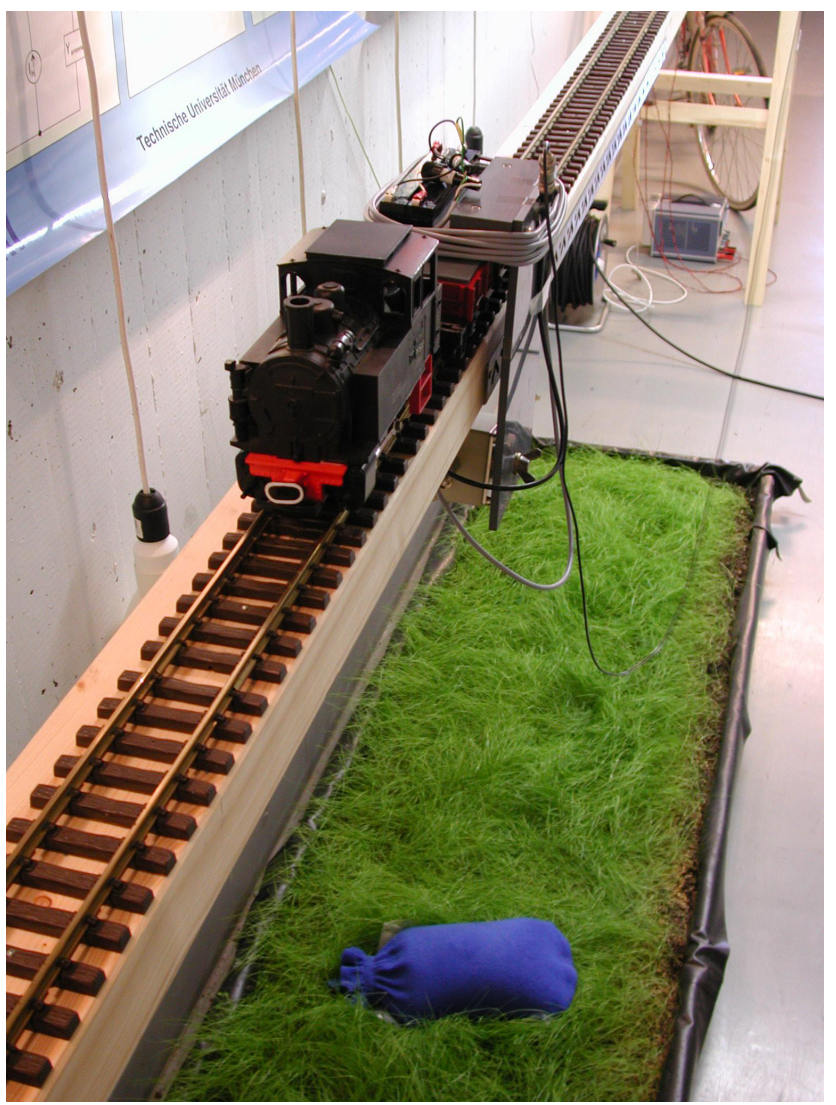

Fig. 7. Laboratory setup for evaluation of the performance of microwave sensor and signal processing.
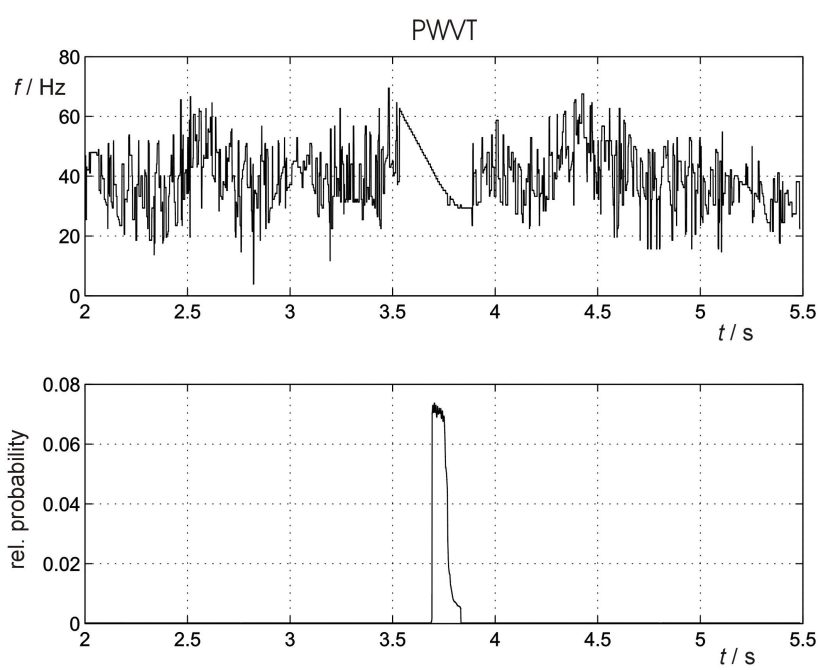

Fig. 8. Typical time-frequency and detection probability response obtained from the hot-water bottle (lab model of a fawn).

higher than necessary in order to economize computational resources for real-time analysis. Once the course of the spectral peak over time is calculated, as shown in Fig. 8, the detection algorithm consists of finding the characteristic frequency slope. A straight line is compared to the slope and

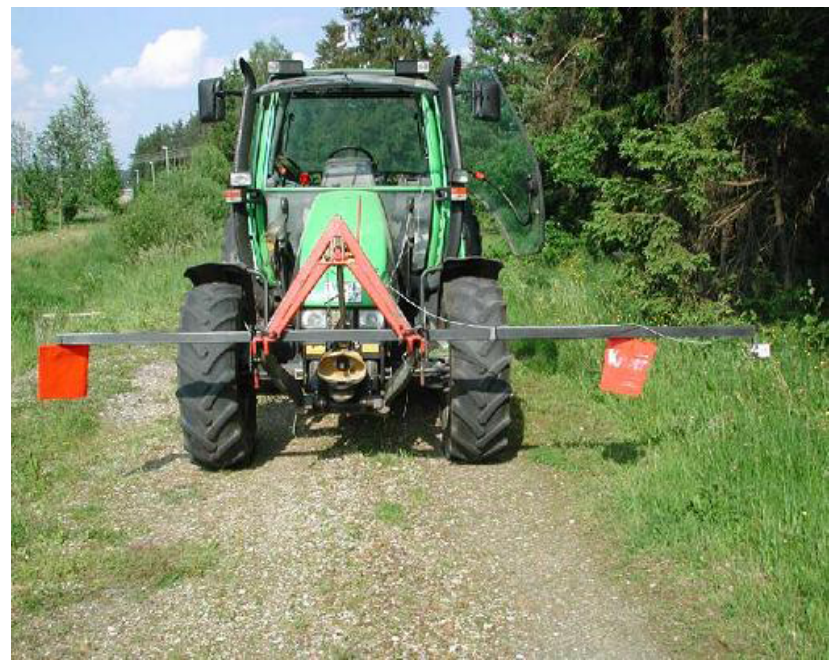

Fig. 9. Microwave sensor mounted at a tractor scanning the natural cover next to a dirt road.
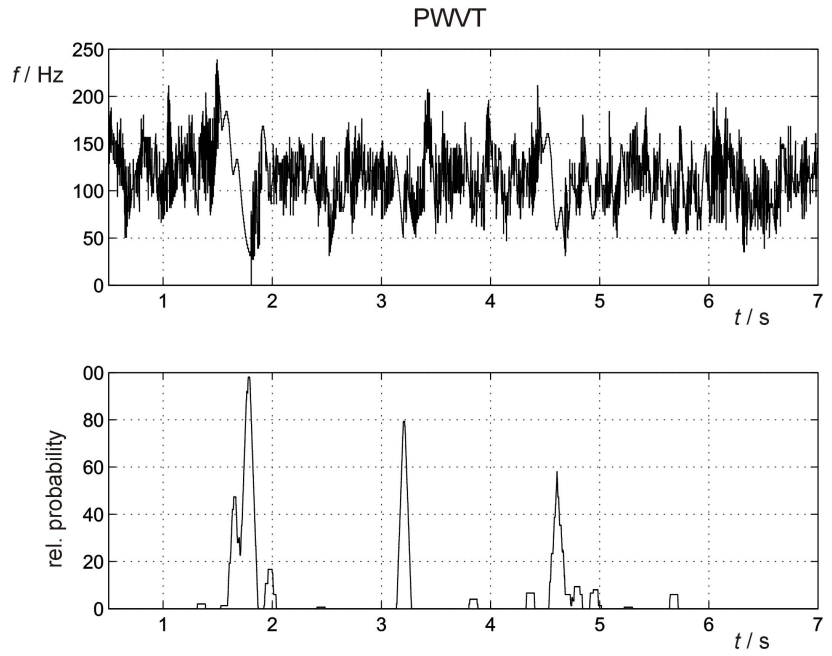

Fig. 10. Time-frequency spectrum obtained from a measurement run over two corner reflectors (at $t=1.8 \mathrm{~s}$ and $t=4.6 \mathrm{~s}$ ) and a hare (at $t=3.2 \mathrm{~s}$ ).

the least square error is calculated. The slope is not always as perfect as shown in Fig. 8. It can be superposed with noise or oscillations, resulting in a big error. If the error is under a predefined limit, the system will indicate the detection of an animal. This error sum and the length of the line, as well as possible limits for its declination, determine the probability of detection or error, respectively.

\section{Evaluation in the field}

The microwave detection system was evaluated during two measurement campaigns. In late summer, the sensor was mounted at a tractor scanning the sparse natural cover next to a dirt road (Fig. 9). A typical result for detection of a hare is shown in Fig. 10. Two corner reflectors provided reference 

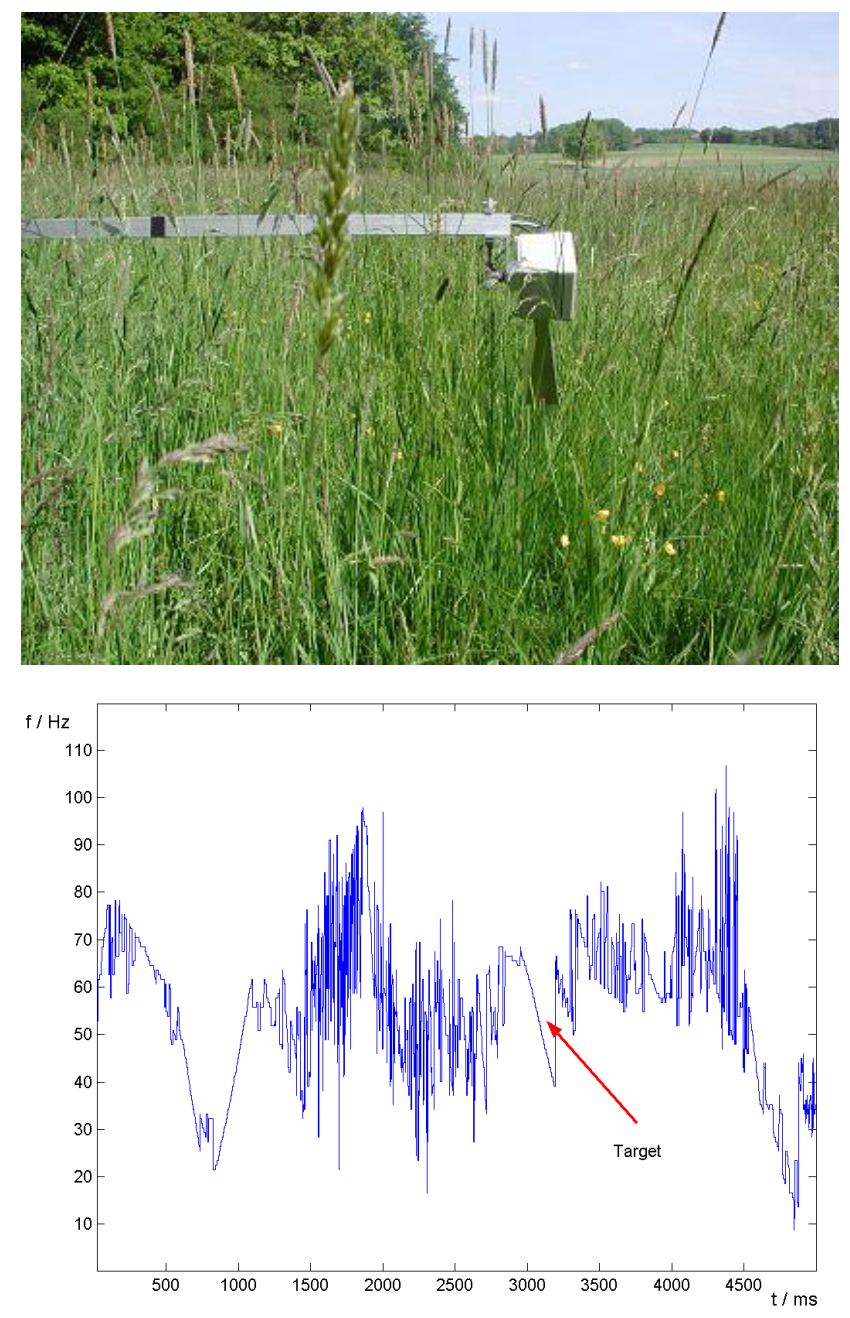

Fig. 11. Measurement of a target in spring-time pasture: (a) Photograph of the sensor above the pasture, (b) measured time-frequency spectrum.

positions. As can be seen, the animal provides a very clear radar response. Due to unevenness of the road, the elasticity of the aluminium bar and the sensor's weight sometimes led to vertical oscillations of the sensor resulting in sinusoidal, low-frequency Doppler signals that disturbed the detection significantly.

During the following spring measurements were made in actual spring-time pasture. In order to prevent the pasture from too much damage the sensor was mounted onto a rotary base. The resulting curved trajectory does not much affect the Doppler signal. Since no fawn could be found in the pasture the hot-water bottle was used as a model. Figure 11 shows the picture of typical spring-time pasture and the respective measured time-frequency spectrum. As can be seen, the target is reliably detected. As similar result was obtained for a target completely covered by the pasture, i.e. invisible for all optical detectors.

Finally, the target was placed beneath a dense cover of thick, herbaceous plants (Fig. 12). Even under this
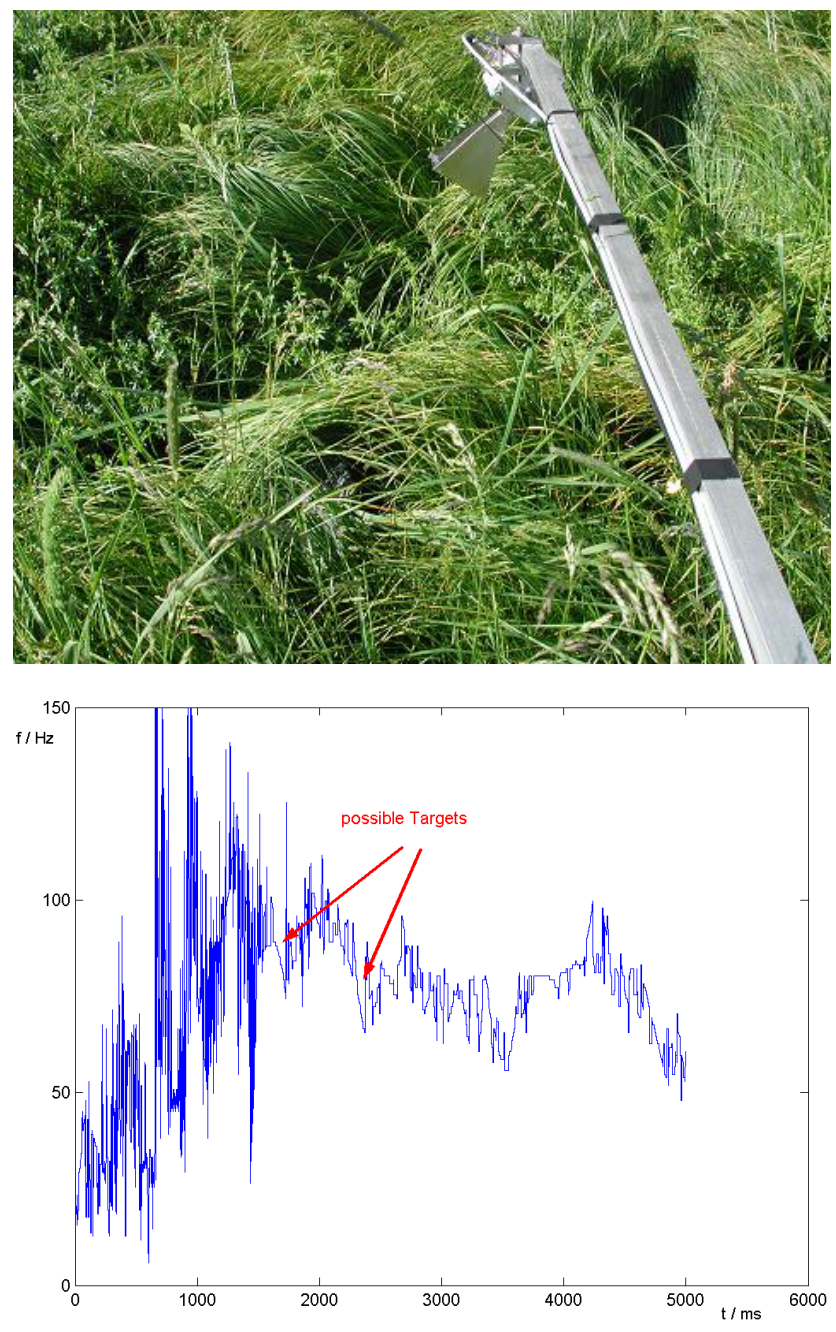

Fig. 12. Measurement of a target beneath a thick cover of herbaceous plants: (a) Photograph of the sensor above the pasture, (b) two measured time-frequency spectra.

"worst-case" condition, a detection reliability of about $50 \%$ was obtained.

\section{Conclusion}

A microwave sensor based on a commercial low-cost $24 \mathrm{GHz}$ Doppler radar module was employed for detection of animals in spring-time pasture. The detection algorithm is based on a nonlinear pseudo-Wigner-Ville-transformation. A very high reliability of detection was achieved in the laboratory as well as in actual spring-time pasture. Even heavily covered targets could be detected with satisfactory probability. The measurements showed that the coherent detection method (i.e. evaluation of the Doppler signal) is sensitive to vertical oscillations that cannot completely avoided when mounted at a mowing machine. One solution would be to measure these oscillations and to pre-process the signal before performing the nonlinear transformation or to use a non-coherent detection principle like reflectometry or radiometry. 
Acknowledgements. The authors appreciate the help and continuing support by R. Nitsche, ISA GmbH, Weiden, and V. Tank, DLR, Oberpfaffenhofen, Germany.

\section{References}

Biebl, E. M.: Millimeter Wave systems based on SIMMWICs, in: 1999 URSI General Assembly Digest, (Toronto, Canada), 235, 13.-21. Aug. 1999.

Cohen, L.: Time-Frequency Analysis, New Jersey: Prentice-Hall, ch. 4, 1995.

Haschberger, P., Bundschuh, M., and Tank, V.: Infrared sensor for the detection and protection of wildlife, Opt. Eng. 35(3), 882889, 1996.

Rasshofer, R. H. and Biebl, E. M.: Advanced millimeterwave speed sensing system based on low-cost active integrated antennas, in: 1999 IEEE MTT-S Int. Microwave Symp. Digest, (Anaheim, CA), 285- 288, 13.-19. June 1999.

Quian, S. and Chen, D.: Joint Time-Frequency Analysis, New Jersey, Prentice-Hall, 1996. 